\title{
Enjeux, richesses et limites d'un fonds de chansons bretonnes méconnu
}

Éva Guillorel

\section{(2) OpenEdition}

1 Journals

Édition électronique

URL : https://journals.openedition.org/clo/220

DOI : $10.4000 /$ clo. 220

ISSN : 2266-1816

Éditeur

INALCO

\section{Édition imprimée}

Date de publication : 1 janvier 2008

Pagination : 213-229

ISBN : 978-2-85831-181-1

ISSN : 0396-891X

\section{Référence électronique}

Éva Guillorel, «Enjeux, richesses et limites d'un fonds de chansons bretonnes méconnu », Cahiers de littérature orale [En ligne], 63-64 | 2008, mis en ligne le, consulté le 07 juillet 2021. URL : http:// journals.openedition.org/clo/220 ; DOI : https://doi.org/10.4000/clo.220

Ce document a été généré automatiquement le 7 juillet 2021.

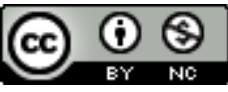

Cahiers de littérature orale est mis à disposition selon les termes de la Licence Creative Commons Attribution - Pas d'Utilisation Commerciale 4.0 International. 


\title{
Enjeux, richesses et limites d'un fonds de chansons bretonnes méconnu
}

\author{
Éva Guillorel
}

1 Comment réunir en peu de temps et avec peu de moyens une importante collection de chansons de tradition orale ? En 1906, le jeune vicaire breton Jean-Marie Perrot répond à cette question en organisant un vaste concours portant sur le répertoire du Léon (Nord-Finistère). En incitant des contributeurs individuels à lui envoyer les chansons qu'ils connaissent ou qu'ils peuvent recueillir auprès de leurs proches, il rassemble ainsi plus de cinq cents pièces en deux mois. Après plusieurs rééditions du concours durant les années qui précédent la Première Guerre mondiale, son fonds atteint plus de mille cent documents, toujours inédits à ce jour ${ }^{1}$. Malgré le souhait de Jean-Marie Perrot de réunir un répertoire semblable à celui qu'ont publié les folkloristes du $\mathrm{XIX}^{\mathrm{e}}$ siècle dans d'autres parties de la Bretagne, le contenu de son fonds, connu sous le nom de Barzaz Bro-Leon ${ }^{2}$, se différencie au contraire très largement de ces références antérieures. Cet écart s'explique avant tout par le mode de constitution du fonds: le recours à un concours permet de réunir directement les contributions des chanteurs, sans passer par l'intermédiaire de collecteurs. Cette complète inversion des perspectives, qui laisse place au regard que les interprètes eux-mêmes portent sur leur répertoire, conduit à s'interroger sur les enjeux, les richesses et les limites d'une telle approche. Plus largement, elle incite à réfléchir aux méthodes et aux sources qui ont fondé la recherche ethnologique sur la chanson de tradition orale, de même qu'aux orientations mises en œuvre dans le travail d'enquête orale, dans un domaine qui dépasse largement les frontières de la Bretagne. 


\section{L'ORIgINALITÉ D'UN CONCOURS DE CHANSONS DE TRADITION ORALE DE 1906}

2 Envisager une collecte de chansons de tradition orale sous la forme d'un concours est une entreprise très novatrice en 1906. Aucune autre collecte de chansons de vaste ampleur n'a été réunie de cette façon en Bretagne ou dans l'ensemble de la France. On peut, certes, citer hors de Bretagne, le concours plus tardif lancé par Jean Baucomont au sujet du folklore enfantin en 1931-1932, mais il concerne les formulettes et non les chansons. La collection réunie par Victor Smith dans le Forez contient également quelques cahiers rédigés par les chanteurs eux-mêmes, mais ce n'est pas un concours : le collecteur rémunérait ses informateurs pour ce travail de mise par écrit de leur répertoire. Entre les années 1938 et 1943, le journaliste Joseph Thomas LeBlanc réunit quant à lui une importante collection de chansons franco-canadiennes après avoir incité les lecteurs du journal acadien L'Évangéline à lui adresser le répertoire qu'ils connaissent (Cormier et Deschênes, 1991); mais il ne s'agit pas non plus d'un concours ${ }^{3}$. Pour une période plus récente encore et en Italie cette fois - mais concernant un répertoire pour une bonne part en langue française - on peut mentionner le concours Cerlogne qui invitait, en 1980-1981, à recueillir des chants de la Vallée d'Aoste dans le cadre d'enquêtes menées par les écoles ${ }^{4}$. Mais ces exemples restent peu nombreux et le Barzaz Bro-Leon n'a pas, à son époque, d'autre équivalent.

3 Le recours à un concours ne fait pourtant que reprendre une forme de divertissement et d'émulation de la culture paysanne qui est particulièrement développée en Bretagne. Jean-Marie Perrot a bien perçu l'engouement de ses compatriotes pour ces jeux, et il réutilise toute sa vie la même technique pédagogique, notamment lorsqu'il prend la direction de la revue bretonnante Feiz ha Breiz en 1907: il s'agit d'intéresser et de stimuler son lectorat par des concours de lecture, d'écriture ou de devinettes (Élégoët, 1976, 56-57). Suite à l'expérience de 1906, des concours de chant, mais aussi de contes ou de jeux paysans sont organisés chaque année au cours de la grande fête culturelle du Bleun Brug ${ }^{6}$, dont Perrot est également l'initiateur. Avant 1906, l'Union Régionaliste Bretonne, fondée en 1898 , organise déjà des concours de chant au cours de ses congrès annuels. Toutefois, ce sont des pièces de composition qui sont lauréates ${ }^{7}$, même si certaines chansons de tradition orale collectées par Jean-Louis Larboulette à Plouhinec sont primées dans la catégorie «mélodies bretonnes » au congrès de 1904 à Gourin. Une incitation à la collecte de termes de vocabulaire breton qui ne se trouvent pas dans les dictionnaires, d'informations sur les vieux métiers, ou encore un petit concours récompensant les meilleures descriptions des dévotions aux saints anciens de Bretagne trouvent eux aussi déjà place dans Feiz ha Breiz avant le lancement du Barzaz Bro-Leon ${ }^{8}$. Jean-Marie Perrot adapte donc à la chanson de tradition orale une méthode de collecte déjà connue et utilisée de façon accessoire par ailleurs.

4 Le Barzaz Bro-Leon se démarque cependant par son ampleur et par la visée identitaire à laquelle cette entreprise est liée dans l'esprit de son initiateur. Le lancement du concours, à l'été 1906, intervient dans un contexte politique et religieux très tendu, suite à la mise en place d'une politique de lutte anticléricale menée par le gouvernement républicain, qui culmine en 1905 avec la loi de Séparation de l'Église et de l'État. C'est dans ce cadre troublé que Jean-Marie Perrot décide de s'investir au service de la défense et de la revivification du breton, de la foi et des traditions 
léonardes. L'initiative du Barzaz Bro-Leon constitue l'une des multiples facettes de cet engagement, qu'il poursuit tout au long de sa vie (Poisson, 1955).

5 L'annonce du concours paraît le 9 juin 1906 dans les colonnes du Courrier du Finistère, un hebdomadaire catholique bilingue qui constitue le deuxième plus gros tirage départemental et qui touche particulièrement le lectorat paysan (Jézéquel, 1971, 77-84). Il s'agit d'un véritable manifeste en faveur de la richesse de la tradition orale toujours vivante et de la culture bretonne et plus particulièrement léonarde. Le choix du titre de Barzaz Bro-Leon est en lui-même tout un programme ; il fait référence au célèbre BarzazBreiz, recueil de chants publié dès 1839 par Théodore Hersart de La Villemarqué, dont le jeune ecclésiastique affirme vouloir s'inspirer. Jean-Marie Perrot, après avoir fort justement noté qu'aucune collecte de chansons léonardes n'a été jusqu'alors publiée contrairement aux autres grandes régions de Bretagne bretonnante - entend pallier cette lacune et réunir dans un livre les plus belles pièces envoyées au concours. Constatant que les chants anciens se perdent auprès des jeunes générations, il plaide pour une véritable démarche de réoralisation de la tradition orale : le support écrit doit permettre de réapprendre aux plus jeunes le savoir oublié des anciens. Le but final est de régénérer la langue et la culture bretonnes tout en développant une politique d'éducation catholique et populaire.

6 Le texte de présentation générale du concours est suivi de dix articles qui forment le règlement, dans lesquels sont précisés le délai imparti - deux mois - et la nature du répertoire sollicité. Jean-Marie Perrot indique clairement qu'il ne veut pas de chansons imprimées, mais uniquement des pièces de tradition orale recueillies en Léon. En connaisseur, il ne rejette cependant pas les cahiers manuscrits de chansons, qu'il incite au contraire à envoyer tels quels. Cette approche, adaptée à une population léonarde fortement alphabétisée par rapport au reste de la Basse-Bretagne (Roudaut, 1984 : 639-648), est très moderne. Elle s'oppose à la conception des premiers collecteurs $\mathrm{du}$ xIX ${ }^{\mathrm{e}}$ siècle, pour qui l'idée romantique d'une performance purement orale recueillie auprès de milieux illettrés, au sein desquels se seraient transmis les chants de génération en génération depuis de nombreux siècles, exclut le recours à des supports écrits préexistants à la collecte. Ces aide-mémoire ont aussi été souvent négligés par les collecteurs du xxe et du début du xxI siècle, et l'attention de Jean-Marie Perrot sur ce point mérite donc d'être particulièrement soulignée.

7 L'initiateur du concours insiste également sur l'importance de la notation des mélodies. Si cette démarche rejoint celle de la majorité des collecteurs du $\mathrm{xx}^{\mathrm{e}}$ siècle, elle n'a que trop rarement été mise en œuvre au siècle précédent - faute de compétences en la matière - par des folkloristes bretons de renom comme Gabriel Milin, Jean-Marie Penguern, François-Marie Luzel ou Anatole Le Braz.

8 Enfin, le règlement précise que, en plus des prix accordés pour la collecte, qui doivent être remis au cours de la fête du Bleun Brug, d'autres récompenses seront également données pour les meilleures interprétations de chants bretons. Ici encore, Jean-Marie Perrot affirme sa conception d'une culture populaire vivante et conviviale : bien loin des folkloristes du $\mathrm{XIX}^{\mathrm{e}}$ siècle qui agissaient avant tout en collectionneurs de pièces rares, sans souci de favoriser la continuité d'une transmission orale qu'ils considéraient comme étant sur le point de s'éteindre, le jeune vicaire entend faire de la collecte non une fin, mais un moyen de revivifier la tradition orale et, à travers elle, la culture bretonne tout entière. 


\section{UN CONTENU IMPOSANT ET HÉTÉROCLITE}

9 Après les différentes éditions du concours entre 1906 et le début de la Première Guerre mondiale, près de mille cent pièces, envoyées par plus de cent cinquante particuliers, sont rassemblées dans le fonds du Barzaz Bro-Leon tel qu'il est connu aujourd'hui ${ }^{9}$. L'ampleur de cet ensemble est tout à fait remarquable : alors que le Léon est considéré depuis la seconde moitié du XIXe siècle comme le parent pauvre des pays de BasseBretagne en matière de tradition chantée et qu'il souffre de nombreux préjugés négatifs à son égard, le résultat du concours de 1906 permet de prouver que cette région jusqu'alors sous-prospectée possède elle aussi un riche répertoire.

10 Mais c'est surtout la diversité des envois qui est l'aspect le plus marquant de cette collection. Le règlement du concours stipule clairement que seules des chansons de tradition orale en langue bretonne et recueillies en Léon sont recherchées. Dans les faits, le fonds contient une grande variété de genres littéraires, qui ne sont pas tous issus du répertoire de tradition orale, qui sont pour certains en français, et dont la provenance dépasse parfois nettement les frontières du Léon. En cela, le Barzaz Bro-Leon se distingue profondément de toutes les publications de folkloristes au XIX ${ }^{\mathrm{e}}$ siècle, qui sont souvent caractérisées par une remarquable homogénéité : ce sont avant tout les complaintes tragiques présumées anciennes - ou gwerzioù - et les chansons d'amour ou au contenu plus léger - sonioù - qui sont recherchées et publiées. Dans le fonds de JeanMarie Perrot, au contraire, des chansons de tous styles et de toutes époques côtoient tous les autres genres de la tradition orale (contes, formulettes, proverbes, comptines, devinettes, monologues et anecdotes). On relève également quelques prières, une petite pièce de théâtre, un sermon ou encore de courts essais littéraires sur des sujets divers.

11 Les chansons correspondent cependant à la très grande majorité des envois, puisqu'elles constituent $89 \%$ des pièces contenues dans le fonds, soit neuf cent quatrevingt-neuf versions. Les gwerzioù de style ancien sont très faiblement représentées en comparaison avec les collectes plus anciennes, puisqu'elles ne forment que $5 \%$ des chansons. Les sonioù sont plus présentes $(23 \%)$, mais l'originalité du fonds tient à la place qu'occupent le répertoire profane dans le style des feuilles volantes (18\%) et surtout les compositions récentes au style imité des canons esthétiques et de la métrique lettrée d'inspiration française en vogue au tournant $\mathrm{du} \mathrm{xx}^{\mathrm{e}}$ siècle, qui présentent peu ou pas de marques de folklorisation $(39,5 \%)^{10}$. Ces deux dernières catégories sont pour ainsi dire totalement absentes des collectes manuscrites et encore plus imprimées au XIX $X^{e}$ siècle.

12 Le Barzaz Bro-Leon apparaît en fait comme un véritable chaînon manquant qui permet de comprendre l'évolution et le renouvellement du répertoire breton au tournant du $\mathrm{xx}^{\mathrm{e}}$ siècle. Il contient à la fois des chants anciens à l'état de bribes, que l'on retrouve massivement dans les collectes $\mathrm{du} \mathrm{XIX}^{\mathrm{e}}$ siècle, et un répertoire plus récent, correspondant à celui qui est souvent recueilli au cours des enquêtes postérieures et encore largement entendu aujourd'hui auprès des informateurs léonards les plus âgés. 


\section{L'INFLUENCE DU MODE DE CONSTITUTION SUR LE CONTENU DU FONDS}

13 Cette singulière diversité des pièces contenues dans le Barzaz Bro-Leon s'explique largement par le mode de constitution du fonds. Le recours à un concours induit un grand nombre de contributeurs qui envoient leur répertoire sans concertation les uns avec les autres. Surtout, il invite les chanteurs eux-mêmes à envoyer leurs chansons, là où les enquêtes orales du XIx ${ }^{e}$ siècle se font par l'intermédiaire d'un collecteur le plus souvent issu d'un milieu social autre que celui de ses informateurs, qui choisit, trie et classe les pièces qu'il entend pour ne retenir que celles qui correspondent à ses attentes. L'énorme écart entre le résultat obtenu par le biais de ces deux démarches conduit à s'interroger sur la pertinence et la représentativité des collectes des XIX et $\mathrm{xx}^{\mathrm{e}}$ siècles par rapport à l'ensemble du répertoire chanté.

14 Les collectes des folkloristes bretons du XIX siècle contiennent en effet avant tout des gwerzioù. Ce souci de recueillir des complaintes anciennes à caractère historique trouve sa naissance dans le mouvement romantique européen de valorisation de la culture et de la mémoire populaires. Pour les premiers collecteurs, la chanson comporte avant tout un intérêt historique et même archéologique. Le chevalier de Fréminville intègre ainsi plusieurs gwerzioù dans ses Antiquités du Finistère et ses Antiquités des Côtes-du-Nord ; les références historiques sont omniprésentes dans le Barzaz-Breiz de La Villemarqué ; quant à Jean-Marie de Penguern, il s'intéresse autant aux chansons qu'à la numismatique ou aux fouilles archéologiques.

Dans cet état d'esprit, il n'est pas étonnant que les folkloristes aient trié, parmi le répertoire qu'ils entendaient, les chansons qu'ils ont notées. Orientant les informateurs pour obtenir avant tout des grandes complaintes, ils n'écrivent ensuite pas tous les textes qu'ils entendent, en fonction de leur opinion de ce qu'est une «belle chanson ». Narcisse Quellien a sur cette question un avis clairement tranché, dont il fait part dans son Rapport de mission en Basse-Bretagne, imprimé en 1883 :

C'était à qui ne savait plus chanter ou n'avait rien dans la mémoire, sinon des refrains et quelques couplets connus de tout le monde. Rien : car je compte pour rien ces sônes modernes, des bagatelles sans la moindre poésie ${ }^{11}$.

16 Outre les compositions récentes, les chansons en français sont également très largement ignorées par les folkloristes, alors qu'elles constituent une réalité du répertoire de Basse-Bretagne ${ }^{12}$. La date présumée et le style des chansons ne sont pas les seuls critères de sélection: le thème des pièces entre également en jeu. Il est frappant de noter la complète absence du répertoire de chansons grivoises dans les collectes publiées, mais également dans les carnets d'enquête des folkloristes. Dans ce cas, on peut penser que, face à ces «messieurs » qui venaient noter leur répertoire, les chanteurs-paysans ont d'eux-mêmes autocensuré leur répertoire en ne choisissant aucune pièce qui puisse sembler inconvenante : la médiation de l'enquêteur, surtout dans le contexte du collectage largement aristocratique du xIx ${ }^{e}$ siècle, apporte ici une orientation particulièrement marquée. D'autres pièces, notamment liées au clergé, ont également fait l'objet d'une autocensure : plusieurs d'entre elles ont bien été notées par certains folkloristes, mais n'ont pas été publiées ${ }^{13}$.

Outre le choix des pièces recueillies, une normalisation des textes est réalisée par les enquêteurs. Largement critiquée chez La Villemarqué, elle est également une réalité - 
certes moins marquée - chez les collecteurs considérés comme les plus scrupuleux. Luzel lui-même, qui constitue encore aujourd'hui la référence en matière de collecte de chansons bretonnes au XIX ${ }^{\mathrm{e}}$ siècle, a modifié des textes pour harmoniser le nombre de syllabes et de vers par strophes, bretonnisé du vocabulaire, ou encore découpé les chansons en parties numérotées pour en faciliter la lecture ${ }^{14}$.

Enfin, le classement opéré par les collecteurs conduit à une homogénéisation du répertoire. La Villemarqué propose dans son Barzaz-Breiz une répartition des pièces en trois grands ensembles: Chants mythologiques, héroïques, historiques et ballades; chants de fêtes et chants d'amour; légendes et chants religieux. À cette division ternaire répond la répartition binaire de Luzel entre gwerzioù et sonioù, très souvent reprise par la suite.

En comparaison avec les collectes des folkloristes, le très hétéroclite Barzaz Bro-Leon semble offrir une image plus proche de la réalité et de la diversité du répertoire chanté en 1906. La forme du concours présente le grand avantage de s'adresser directement aux chanteurs et ainsi de supprimer la médiation du folkloriste, et avec elle un certain nombre de présupposés et d'orientations propres à sa démarche et au contexte dans lequel il évolue. Il n'est pas étonnant, dès lors, de constater l'importante place qu'occupe dans le Barzaz Bro-Leon le répertoire issu des chansons sur feuilles volantes et, de manière encore plus marquée, les compositions très récentes qui connaissent alors leur heure de gloire. Les chanteurs ont envoyé les pièces qui, selon le goût de leur époque et de leur classe sociale, sont les plus belles, c'est-à-dire justement celles qui sont rejetées par les folkloristes comme étant sans valeur. Une anecdote notée par Anatole Le Braz dans l'un de ses carnets d'enquêtes révèle bien cette profonde incompréhension entre le goût populaire et le goût des folkloristes. La situation se déroule en 1890 à Penvénan, alors qu'il note des chansons au cours d'une veillée :

Izabelle Bellec, femme de Corniou, a une voix très forte et très juste. Elle commence par me chanter une gwerz récente d'un soldat qui a été combattre les Bédouins en Afrique et qui, venant loger dans une hôtellerie, qu'il ne sait pas tenue par sa mère, est assassiné par celle-ci, parce qu'il rapporte de l'argent. Je lui dit [sic] qu'elle est imprimée, d'en entreprendre une autre; mais aussitôt tout l'auditoire proteste. Tout ce monde aime autant que jamais le chant et la gwerze bretonne : Lezt ann-hei da ganan, me crie-t-on, ma clewfomp ar peur-rest ${ }^{15}$. »

L'absence de classement du fonds par catégories de chansons est également précieuse, dans la mesure où elle évite le processus de normalisation arbitraire qui découle du découpage du répertoire entre gwerzioù et sonioù. Le Barzaz Bro-Leon n'est pas rangé par catégories thématiques ou par genres, sans pour autant composer un ensemble déclassé et sans ordre interne. La conservation des envois par communes d'origine a permis de garder la logique initiale du fonds, qui est d'ordre géographique. Aucune collecte basbretonne publiée depuis le xix ${ }^{e}$ siècle n'a opéré un tel choix. L'absence de classement thématique permet en outre, au sein de chaque commune, de conserver en un seul ensemble le répertoire d'un chanteur. L'envoi d'Ouessant rassemble ainsi les cinquantesix pièces adressées par une unique informatrice. Là encore, aucune importante collecte bretonne publiée n'a eu le souci de garder de façon groupée le répertoire des différents chanteurs, même si quelques monographies autour d'un seul interprète ont été réalisées au cours des dernières décennies ${ }^{16}$. Cette disposition donne pourtant l'avantage de mettre en évidence les sensibilités de certains interprètes, lisibles à travers le répertoire qu'ils connaissent et qu'ils ont choisi d'envoyer au concours. 
21 Les chansons du Barzaz Bro-Leon présentent également l'immense richesse d'avoir été écrites dans une langue et une orthographe non normalisées, reflet des tournures de phrase et des prononciations dialectales des chanteurs. Certaines d'entre elles, écrites par des contributeurs manifestement peu familiers du breton écrit, révèlent une langue notée de manière largement phonétique, exempte de ponctuation et de majuscules.

\section{CONCOURS ET COLLECTE : LES LIMITES}

Les spécificités tout à fait remarquables du Barzaz Bro-Leon ne doivent cependant pas conduire à surestimer l'intérêt de ce fonds en le considérant comme un moyen de connaître le "vrai » répertoire chanté et d'avoir un accès direct à un discours entièrement "populaire ». Le choix d'une annonce dans un journal implique déjà un certain public. En effet, loin d'atteindre l'ensemble des chanteurs léonards, ce concours touche avant tout la couche la plus aisée de la paysannerie, celle qui maitrise la lecture et l'écriture et qui est abonnée à un hebdomadaire. C'est aussi celle dont le goût a été influencé par les productions écrites en breton et en français et par la mode des compositions récentes des poètes et des chansonniers locaux. Même si, à partir d'un lecteur, la nouvelle peut ensuite se diffuser oralement, il est évident qu'une partie des chanteurs n'a pas entendu parler de ce concours ou ne s'y est pas intéressé. Parmi ceux-ci, il faut vraisemblablement compter une surreprésentation des populations les plus âgées, les moins intégrées et les moins alphabétisées, celles-là mêmes, dont le répertoire de tradition orale peut être particulièrement riche et peu influencé par l'imprimé.

Les termes utilisés dans l'annonce du concours constituent quant à eux une médiation, à la manière de celle qui est opérée par les collecteurs, et orientent le choix des pièces envoyées. L'appel de 1906 insiste par exemple sur le fait que les chansons imprimées ou que l'on trouve dans les livres ne sont pas concernées par le concours. Or, cette précision est ambiguë, comme le révèle l'extrait d'une lettre envoyée par Joseph Chauvin, de Saint-Pol-de-Léon, à Jean-Marie Perrot en 1907, qui est conservée dans le fonds du Barzaz Bro-Leon :

J'ai recueilli cet hiver quelques chansons que je vous enverrais prochainement si vous le desirez. C'est vraiement difficile de tomber sur des œuvres vraiement originales dans ce pays-ci. A force de courir les veillees et de faire jaser les bonnes gens j'en avais trouve une dizaine qui me semblaient meriter quelque droit à la conservation, mais jugez de ma deception quand l'autre jour Monsieur Conq le vicaire de Saint-Pol m'ayant prêté les Soniou Breiz-Izel de Luzel, je fus bien surpris et un peu ennuyé je vous l'avoue de voir les trouvailles que j'estimais le plus à leur place dans le recueil du Barzaz-Treguer. C'est une deception qui doit arriver je pense à bien d'autres que moi et qui refroidit à merveille les enthousiasmes des chercheurs novices.

On sent ici clairement les limites d'un concours qui sollicite, comme le dit très bien ce contributeur, des " chercheurs novices ", qui ne sont pas habitués à faire de la collecte et qui, pour nombre d'entre eux, ignorent largement les caractéristiques du répertoire chanté de tradition orale. Ces hommes et ces femmes n'ont aucune sensibilité à la démarche ethnologique de collectage, tout simplement parce qu'ils n'y ont jamais été formés, et ils n'ont pas de vue d'ensemble de ce répertoire. D'où une compréhension erronée de l'intention de Jean-Marie Perrot ; là où le vicaire de Saint-Vougay cherche à faire entendre qu'il ne veut pas recevoir de feuilles volantes imprimées ou de textes 
recopiés dans un livre, ses lecteurs comprennent qu'il ne faut pas envoyer de versions de chants-types déjà publiés ailleurs, méconnaissant que la multiplicité des versions d'une même chanson est une caractéristique essentielle de la transmission orale et que la prise en compte de leur diversité est la base de toute démarche d'enquête ethnographique. Ce courrier met ainsi le doigt sur une des faiblesses majeures de cette entreprise et permet d'imaginer la perte engendrée par de tels raisonnements : Joseph Chauvin n'a finalement pas envoyé les versions qu'il mentionne. Ceci dit, il faut minimiser l'impact de cette ambiguïté dans la formulation du concours, dans la mesure où la plupart des lecteurs du Courrier du Finistère n'ont de toute évidence pas consulté les Gwerzioù et Sonioù de Luzel avant d'y participer.

Une autre limite tient dans l'incapacité technique pour beaucoup de contributeurs de noter la mélodie, ainsi que le souhaitait Jean-Marie Perrot. Plusieurs participants du concours s'excusent ainsi par écrit de ne pas être capables de donner l'air de leurs chansons.

Se pose ensuite la question de la part du choix personnel des chanteurs dans le répertoire envoyé. La logique d'un concours incite les participants à retenir et à consigner les pièces qui, selon eux, ont le plus de chances d'être primées. Surtout, ce concours est organisé par un ecclésiastique et les prix sont solennellement remis au cours d'une fête très médiatisée. Dans ce contexte, il est évident que les chanteurs ont usé d'autocensure sur leur propre répertoire. Les chansons gaillardes sont ainsi, sans surprise, presque totalement absentes.

Par ailleurs, si le Barzaz Bro-Leon présente la particularité d'être une collecte sans intervention de folkloristes, des intermédiaires ont tout de même pu participer à la mise par écrit de certaines pièces. Les enfants et petits-enfants, plus alphabétisés que les anciens, sont explicitement sollicités pour apporter leur aide dans la première annonce du concours. Des notables locaux - notamment les instituteurs et les prêtres ont aussi pu être mis à partie dans cette entreprise. Un contributeur de Lannilis précise ainsi dans la lettre qu'il joint à son envoi qu'il est incapable de noter les mélodies, mais que, si le jury du concours trouve ses chansons intéressantes, il pourra demander à un vicaire de la paroisse de les noter pour lui. Ces intermédiaires lettrés minimisent alors l'accès direct au répertoire des chanteurs; en outre, la tendance à l'autocensure de la part des interprètes est de toute évidence plus grande s'ils chantent directement devant un prêtre chargé de noter les paroles par écrit.

Enfin, le principe d'un concours favorise le concept d'envoyeur et non celui d'informateur : peu importe qui a interprété la chanson, l'important étant le nom de la personne qui l'a adressée au jury dans l'espoir d'être lauréat. Cette spécificité du Barzaz Bro-Leon entraîne une large zone de flou sur le statut des contributeurs, qui ne précisent le plus souvent pas s'ils sont eux-mêmes chanteurs, compositeurs ou collecteurs des pièces qu'ils envoient. On sent ici une autre limite de cette collecte, car si la forme du concours permet bien de rassembler très rapidement un grand nombre de pièces provenant de l'ensemble du Léon, cette efficacité se fait au détriment de la qualité des informations au sujet de ce répertoire. Il n'y a là aucune contradiction dans l'esprit du Barzaz Bro-Leon, qui ne se définit pas comme une démarche à visée ethnologique, mais comme une entreprise à vocation culturelle et identitaire. 


\section{L'ETHNOLOgIE SANS ENQUÊTE ? L'APPORT DES LETTRES ENVOYÉES PAR LES CONTRIBUTEURS}

La faiblesse des informations concernant les interprètes est compensée par la présence d'une trentaine de lettres qui sont jointes aux envois et qui constituent là encore une originalité du Barzaz Bro-Leon.

Ces lettres nous informent en premier lieu sur les contributeurs. Deux catégories de correspondants se dégagent, d'une part les amis et connaissances de Jean-Marie Perrot, de l'autre les individus qui participent au concours après en avoir lu l'annonce dans le Courrier du Finistère: le choix de la langue - breton ou français - et la plus ou moins grande aisance dans l'écriture permettent rapidement d'estimer leur statut socioculturel, même si les plus illettrés des participants n'ont certainement pas envoyé de courrier ou ont demandé à un tiers de le rédiger pour eux.

31 Le ton des lettres est, lui aussi tout à fait significatif. Des marques d'amitié pour la première catégorie de correspondants, qui n'hésitent pas à joindre à leurs lettres des nouvelles de leur famille ou de leur entourage, on passe à un ton de déférence et d'humilité parfois émouvant lorsqu'il s'agit de contributeurs de condition modeste qui s'adressent du mieux qu'ils le peuvent à un ecclésiastique.

Les informations les plus intéressantes sur le plan ethnologique sont celles qui concernent les chanteurs, la provenance du répertoire, et les conditions de collecte et de composition des paroles et des airs. On apprend au hasard des lettres que tel participant est cultivateur, telle autre religieuse ou sœur d'un missionnaire parti au Tonkin. Des renseignements plus précis concernent parfois la provenance du répertoire : apprentissage dans le cadre familial ou communautaire, date à laquelle une chanson a été entendue pour la première fois, personnes auprès desquelles un contributeur a recueilli ses chants ou encore liste des complaintes entendues couramment dans les veillées en 1906. La correspondance adressée par la famille de Jean-Marie Perrot à Ploumoguer et à Milizac est pour sa part particulièrement intéressante pour comprendre les réseaux familiaux de collecte au sein desquels évolue le jeune vicaire ${ }^{17}$. Les contributeurs qui composent des pièces originales parlent quant à eux de leurs inspirations et des chansonniers en vogue qu'ils prennent en modèle.

En définitive, les lettres accompagnant le répertoire du Barzaz Bro-Leon apportent une foule de renseignements précieux sur les informateurs, le contexte de collecte ou encore le regard porté par les chanteurs eux-mêmes sur leur répertoire oral, qui ne sont que trop rarement fournis dans les publications des folkloristes du XIX ${ }^{e}$ siècle.

\section{LE DEVENIR DU BARZAZ BRO-LEON APRÈS LE CONCOURS}

Chaque édition du concours se termine par une remise de prix aux contributeurs dont les envois sont jugés les plus intéressants. La liste des lauréats est publiée dans le Courrier du Finistère, de sorte que l'on peut comparer les prix reçus et les envois effectués. Or, on constate que les critères de sélection ne semblent pas toujours coïncider avec la quantité et la qualité des pièces envoyées. Il est sans doute plus juste d'évoquer ici la différence de regard entre les contributeurs, les membres du jury composé de notables locaux laïques et ecclésiastiques - et les ethnologues du début du $\mathrm{XIX}^{\mathrm{e}}$ siècle qui étudient ce fonds a posteriori: chacun de ces trois regards valorise 
différemment les pièces envoyées. Les deux gros cahiers de chansons adressés par Madame Noret, de l'île d'Ouessant, sont particulièrement intéressants de ce point de vue, puisque Jean-Marie Perrot a inscrit au crayon, dans la marge, des appréciations sur la valeur des pièces. Dans ses commentaires positifs, il évoque la pureté de la langue, l'ancienneté présumée des récits, l'originalité de l'intrigue ou encore les valeurs morales et religieuses qui s'en dégagent. D'autres ne trouvent au contraire pas grâce à ses yeux : l'adaptation en breton de la chanson française bien connue du bal sur le pont de Nantes est jugée "sans valeur »; une autre est qualifiée d' "assez vulgaire », sans doute en raison des couplets finaux qui invitent les jeunes filles à boire en l'honneur d'un prisonnier évadé.

L'inadéquation entre les pièces envoyées et primées d'une part, et les souhaits en matière de répertoire qui sont formulés dans l'appel à concourir en 1906 de l'autre, permet également de proposer des explications concernant la non-publication de ce fonds, malgré les promesses réitérées de Jean-Marie Perrot. De toute évidence, les pièces reçues ne correspondent pas à son attente, ce qui peut expliquer son insistance à renouveler des éditions du concours afin de compléter ce qu'il considère comme des manques. En effet, les chansons sont très éloignées du répertoire publié par les folkloristes du XIXe siècle : l'espoir de Jean-Marie Perrot de réunir en Léon, sur le même modèle, le pendant des grandes collectes cornouaillaises, trégoroises et vannetaises a été vraisemblablement déçu. À ces considérations s'ajoutent d'autres circonstances qui ont pu compliquer la publication, notamment le problème de la compatibilité entre ce projet et les orientations de la hiérarchie ecclésiastique de Jean-Marie Perrot, la multiplicité des activités dans lesquelles s'est engagé le jeune vicaire, ainsi que l'irruption de la Première Guerre mondiale.

Dans l'état où il nous est parvenu, le Barzaz Bro-Leon constitue en définitive une pièce majeure au dossier de la genèse des collectes ethnographiques. Son intérêt et sa richesse tiennent au mode original de constitution du fonds : le recours a un concours permet d'avoir accès à un autre regard, celui des interprètes, sur le répertoire chanté en Bretagne au début du $\mathrm{xx}^{\mathrm{e}}$ siècle. La profonde inadéquation entre les fonds recueillis par ce moyen et les collectes individuelles de terrain suscite des questionnements qui dépassent largement les frontières de cette région et qui conduisent à une réflexion plus globale sur les méthodes et les pratiques d'enquête dans le domaine du répertoire de tradition orale.

\section{BIBLIOGRAPHIE}

BERTHOU-BÉCAM, Laurence, 1998, Enquête officielle sur les poésies populaires de la France, 1852-1876. Collectes de langue bretonne, Rennes, Thèse de breton et celtique, inédite.

Centre d'études francoprovençales René Willion de Saint-Nicolas, 1996, Les chants et les chansons valdôtains, Aoste, Musumeci Éditeur. 
CORMIER, Charlotte et DESCHÊNES, Donald, 1991, Joseph-Thomas LeBlanc et le romancéro inachevé, Canadian Folklore Canadien, 13-2, p. 55-70.

ÉLÉGOËT, Fañch, 1979, Prêtres, nobles et paysans en Léon au début du XXe siècle. Notes sur un nationalisme breton : Feiz ha Breiz, 1900-1914, Pluriel, 18, p. 39-90.

FRÉMINVILLE, chevalier de, 1979, Antiquités du Finistère I-II, Paris-Genève-Gex, Slatkine Reprints,

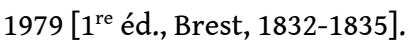

FRÉMINVILLE, chevalier de, 1980, Antiquités des Côtes-du-Nord, Paris-Genève-Gex, Slatkine Reprints

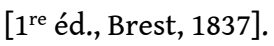

GoYAT, Gilles, 2008, Chansons traditionnelles du pays bigouden. Eur Vigoudenn o kana, Paris, Jouve [1 $1^{\text {re }}$ éd., Brest, 1977].

GuILLOREL, Éva, 2008a, Une expérience inédite de collecte en Bretagne au début du $20^{e}$ siècle : le Barzaz Bro-Leon, Brest, Master 2 d'ethnologie, inédit.

GUILLOREL, Éva, 2008b, La complainte et la plainte. Chansons de tradition orale et archives criminelles : deux regards croisés sur la Bretagne d'Ancien Régime (16 $16^{e} 18^{e}$ siècles), Rennes, Thèse d'histoire, inédite. GUILLOREL, Éva et BOUTHILLIER, Robert, 2009, L'influence des livrets de colportage dans la circulation et le renouvellement du répertoire oral. La destinée de trois complaintes criminelles imprimées en Bretagne au $19^{\mathrm{e}}$ siècle, Bretagnes, du cour aux lèvres. Mélanges en l'honneur de Donatien Laurent, Rennes, Presses Universitaires de Rennes.

JÉzÉQUEL, Jean-Yves, 1971, Le Courrier du Finistère, 1890-1900, Brest, Maîtrise d'histoire, inédite. Kosko, Maria, 1966, Le fils assassiné (AT 939A). Étude d'un thème légendaire, Helsinki, FF Communications 198.

LA Villemarqué, Théodore Hersart de, 1990, Barzaz-Breiz, Paris, Perrin [1 ${ }^{\text {re }}$ éd., Paris, 1839].

LUZEL, François-Marie, 1894, Chansons populaires françaises recueillies en Basse-Bretagne, Revue des Traditions Populaires, IX, pp. 405-410.

LuZEL, François-Marie, 1971, Chants et chansons populaires de la Basse-Bretagne. Soniou I, Paris, Maisonneuve \& Larose, 1971 [ $1^{\text {re }}$ éd., 1890].

Poisson, Henri, 1955, L'abbé Jean-Marie Perrot, fondateur du Bleun Brug (1877-1943), Rennes, Plihon.

QUELLIEN, Narcisse, 1883, Rapport sur une mission en Basse-Bretagne ayant pour objet de recueillir les mélodies populaires, Paris, Imprimerie Nationale.

Rolland, Eugène, 1967, Recueil de chansons populaires, Paris, Maisonneuve \& Larose [1 $1^{\text {re }}$ éd., Paris, 1883-1890].

RoUDAUT, Fañch, 1899, Sones et gwerz couronnées par l'Union Régionaliste Bretonne, Congrès de Vannes de 1899, Vannes, imprimerie Lafolye.

ROUDAUT, Fañch, 1984, La difficile approche de l'alphabétisation de la Basse-Bretagne avant la Révolution, La France d'Ancien Régime. Études réunies en l'honneur de Pierre Goubert, Paris, Privat, p. 639-648. 


\section{NOTES}

1. Une édition critique de ce fonds est en préparation au Centre de Recherche Bretonne et Celtique de l'Université de Bretagne Occidentale.

2. «Poésies du Léon ", en breton.

3. Je tiens à remercier Georges Delarue, Robert Bouthillier et Francine Brunel-Reeves pour leurs précisions.

4. Le résultat a été publié dans : Les chants et les chansons valdôtains (1996).

5. « Foi et Bretagne».

6. «Fleur de Bruyère ». Cette association a pour préoccupation la défense de la foi catholique et de la culture bretonne.

7. Les pièces primées lors du congrès de 1899 sont publiées dans Sones et gwerz couronnées par l'Union Régionaliste Bretonne (1899). L'annonce du lancement du concours de 1907 prévoit pour sa part des prix pour les meilleures gwerzioù, sonioù, mais aussi compositions en prose, pièces de théâtre, vies de saints ou encore essais agricoles. Ar Vro, mars 1907, 10-11.

8. Feiz ha Breiz, mars-avril 190, 63-64.

9. Fonds privé, collection Caouissin.

10. Le reste du fonds est composé de berceuses et chansonnettes enfantines, de cantiques, ou encore de chansons en français (issues ou non du répertoire de tradition orale).

11. Anatole Le Braz tient un semblable discours dans l'introduction de Luzel (1891, XVI-XVII).

12. François-Marie Luzel montre plus d'ouverture en publiant en 1894 un court article sur les chansons en français qu'il a recueillies en Bretagne bretonnante. À la même époque, Eugène Rolland publie également quelques textes et musiques de chansons françaises de Basse-Bretagne. 13. C'est particulièrement vrai pour François-Marie Luzel, qui envoie tout de même plusieurs de ces pièces dans le cadre de l'enquête sur les Poésies Populaires de la France (Berthou-Bécam, 1998, 346-370).

14. La confrontation entre ses carnets d'enquêtes et ses publications en livre d'innombrables exemples. L'un d'entre eux est analysé dans Guillorel (2008b, 213-220).

15. «Laissez-là chanter, que nous entendions la suite », CRBC, Fonds Le Braz, carnet EA, p. 116-117. La gwerz reprend l'histoire du fils assassiné, bien connue dans le répertoire de colportage européen depuis le XVII ${ }^{\mathrm{e}}$ siècle. Bien que cette chanson soit largement attestée dans le répertoire en breton, elle n'est pas recensée dans l'étude de Maria Kosko (1966). Au sujet des versions en breton, voir Guillorel et Bouthillier (2009).

16. Par exemple autour du répertoire de Catherine Madec, de Plozévet (Goyat, 2008).

17. Plusieurs cahiers écrits de la main de Jean-Marie Perrot et conservés dans le fonds du Barzaz Bro-Leon attestent que cet ecclésiastique a lui-même fait de la collecte dans sa paroisse d'origine dès 1902 au moins.

\section{INDEX}

Mots-clés : chanson, concours, répertoire, hétérogénéité

Keywords : Brittany (Léon), Songs of Oral Tradition, Directory, Heterogeneity, Anthropology

Index géographique : Bretagne (Léon)

Thèmes : anthropologie (Europe) 